\title{
Antidepressive Effekte von Vortioxetin in einer Metaanalyse von elf Studien bestätigt
}

Fragestellung: Wie wirksam ist Vortioxetin in der Behandlung depressiver Störungen?

Hintergrund: Vortioxetin, das in Deutschland unter dem Namen Brintellix ${ }^{\otimes}$ eingeführt wurde, ist ein hochpotenter selektiver Serotonin-Wiederaufnahmehemmer (SSRI), der zusätzlich antagonistisch an $5-\mathrm{HT}_{1 \mathrm{D}^{-}}, 5-\mathrm{HT}_{3}$ - und $5-\mathrm{HT}_{7}$-Rezeptoren, partialagonistisch an $5-\mathrm{HT}_{1 \mathrm{~B}}$ - sowie agonistisch an $5-\mathrm{HT}_{1 \mathrm{~A}}$-Rezeptoren wirkt. Es zeigt keine anticholinergen oder antihistaminergen Nebenwirkungen. Die wichtigsten Nebenwirkungen sind auf die Wiederaufnahme von Serotonin zurückzuführen und bestehen damit in Übelkeit und Erbrechen, Schwitzen sowie Durchfall. Ziel der Untersuchung war, die Wirksamkeit von Vortioxetin gegen Placebo nachzuweisen und zu untersuchen, ob eine Dosis-Wirkungs-Be-
Thase ME, Mahableshwarkar AR, Dragheim $M$ et al. A metaanalysis of randomized, placebo-controlled trials of vortioxetine for the treatment of major depressive disorder in adults. Eur Neuropsychopharmacol 2016; 26: 979-93 ziehung $(5-20 \mathrm{mg} / \mathrm{Tag})$ besteht.

Patienten und Methodik: Die Autoren schlossen elf randomisierte placebokontrollierte Studien zur Behandlung von Depressionen über sechs bis acht Wochen mit Vortioxetin in eine Metaanalyse ein. Hauptzielparameter war die Veränderung des Punktwertes auf einer etablierten Depressionsskala, der Montgomery-Åsberg Depression Rating Scale (MADRS). Sekundäre Endpunkte waren Veränderungen auf der Einzel-Item-Ebene der MADRS, die Responserate (50\% Reduktion vom Baseline MADRS), die Remissionsrate (MADRS < 10) sowie der „Clinical Global Impression“-Punktwert. Zwei der fünf Autoren sind bei der Herstellerfirma (Lundbeck) angestellt.

Ergebnisse: In den elf Studien wurden 1.824 Patienten mit Placebo und $3.304 \mathrm{mit}$ Vortioxetin ( $5 \mathrm{mg} / \mathrm{Tag}: \mathrm{n}=1.001,10 \mathrm{mg} /$ Tag: $\mathrm{n}=1.042,15 \mathrm{mg} /$ Tag: $\mathrm{n}=449 ; 20 \mathrm{mg} /$ Tag: $\mathrm{n}=812$ ) behandelt. Die Metaanalyse zeigte, dass Vortioxetin in einer Dosis von 5,10 und $20 \mathrm{mg} / \mathrm{Tag}$ (aber nicht $15 \mathrm{mg}$ ) zu einer signifikanten Reduktion des MADRS-Punktwertes im Vergleich zu Placebo führte $(-2,27,-3,57$ bzw. $-4,57 ; \mathrm{p}<0,01)$. Tagesdosen von 10 und $20 \mathrm{mg}$ waren assoziiert mit signifikanten Verbesserungen in zehn von zehn MADRS Einzel-Items. Vortioxetin führte darüber hinaus zu signifikant höheren Response- und Remissionsraten im Vergleich zu Placebo.

Schlussfolgerungen: Die Autoren schlussfolgern, dass Vortioxetin zur Behandlung von Depressionen wirksam ist und die Behandlungseffekte mit höheren Dosen zunehmen.

\section{- Kommentar von Klaus Lieb, Mainz}

\section{Wirksam, aber ohne Zusatznutzen}

Lundbeck hat mit Citalopram beziehungsweise Escitalopram seit Jahren einen sehr gut wirksamen SSRI auf dem Markt, der allerdings zuletzt im Hochdosisbereich wegen QT-Zeit-Verlängerungen Probleme machte. Mit Vortioxetin wollte man versuchen, wieder einen ebenso erfolgreichen SSRI auf den Markt zu bringen. Das ist insofern gelungen als die Wirksamkeit gegenüber Placebo belegt werden konnte (auch wenn die DosisWirkungs-Beziehung nicht wirklich überzeugt). Das Problem ist nur, dass Lundbeck das IQWiG im letzten Jahr bezüglich eines Zusatznutzens der Substanz nicht überzeugen konnte. Die von Lundbeck vorgelegten Studien waren fast alles placebokontrollierte Studien. In einer weiteren Studie zeigten sich $20 \mathrm{mg}$ Vortioxetin ebenso wie $60 \mathrm{mg}$ Duloxetin Placebo überlegen, und in einer Studie war Vortioxetin Agomelatin überlegen. Was fehlte waren Vergleichsstudien zu SSRI (von denen allerdings zu erwarten gewesen wäre, dass keine Überlegenheit nachgewiesen werden kann, da Vortioxetin im Wesentlichen ein SSRI ist). Die Firma hat daher entschieden, Vortioxetin in Deutschland vom Markt zu nehmen, da infolge der drohenden Eingruppierung in eine Festbetragsgruppe die Erlöse zu niedrig gewesen wären. Das Beispiel zeigt, wie schwie-

rig es ist, neue und bessere Antidepressiva zu entwickeln und auf den Markt zu bringen. Die Forschungsbemühungen der Firmen werden durch solche IQWiG-Entscheidungen in nachvollziehbarer Weise nicht befördert, auf der anderen Seite kann es aber auch nicht im Sinne der Patienten sein, neue Substanzen einzusetzen, deren wirkliches Risiko noch niemand kennt, wenn etablierte Substanzen mit sehr wahrscheinlich gleicher Wirksamkeit und bekanntem Risikoprofil (z. B. Sertralin und Citalopram) seit Jahren auf dem Markt sind.

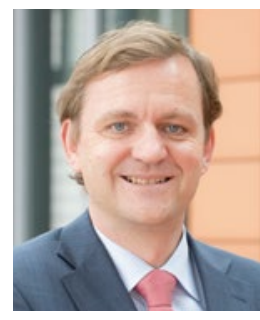

Prof. Dr. med. Klaus Lieb, Mainz 